\title{
BMJ Open Effectiveness and cost-effectiveness of the Salut Programme: a universal health promotion intervention for parents and children-protocol of a register-based retrospective observational study
}

\author{
Inna Feldman, ${ }^{1}$ Eva Eurenius, ${ }^{2}$ Jenny Häggström, ${ }^{3}$ Filipa Sampaio, ${ }^{1}$ \\ Marie Lindkvist, ${ }^{2,3}$ Anni-Maria Pulkki-Brännström, ${ }^{2}$ Anneli Ivarsson ${ }^{2}$
}

To cite: Feldman I, Eurenius E, Häggström J, et al. Effectiveness and costeffectiveness of the Salut Programme: a universal health promotion intervention for parents and childrenprotocol of a register-based retrospective observational study. BMJ Open 2016;6: e011202. doi:10.1136/ bmjopen-2016-011202

- Prepublication history for this paper is available online. To view these files please visit the journal online (http://dx.doi.org/10.1136/ bmjopen-2016-011202).

Received 19 January 2016 Revised 10 June 2016 Accepted 14 July 2016

\section{(a) CrossMark}

${ }^{1}$ Department of Women's and Children's Health, Uppsala University, Uppsala, Sweden ${ }^{2}$ Department of Public Health and Clinical Medicine, Epidemiology and Global Health, Umeå University, Umeå, Sweden

${ }^{3}$ Department of Statistics, USBE, Umeå University, Umeå, Sweden

Correspondence to

Dr Inna Feldman;

inna.feldman@kbh.uu.se

\section{ABSTRACT}

Introduction: There is inadequate evidence for the effectiveness and cost-effectiveness of health promotion interventions. The Salut Programme aims to reach all parents and children in the Västerbotten County of Sweden with a combination of health promotion interventions initiated during pregnancy and continued over the childhood period. This study protocol describes an effectiveness study and an economic evaluation study, where the ongoing Salut Programme is compared to care-as-usual over the periods of pregnancy, delivery and the child's first 2 years of life.

Methods: A register-based retrospective observational study design will be used with existing data sources with respect to exposures and outcomes. Outcomes of interest are clustered at 3 points: around the child's birth, 1 month after the child's birth and 2 years after the child's birth. We will simulate an experiment by retrospectively identifying and comparing children and their parents in the geographical areas where the Salut Programme was implemented since 2006 and onwards, and the areas where the Programme was not implemented before 2009. Outcomes will be analysed and compared for the premeasure period, and the postmeasure period for both groups. Our analysis combines difference-in-difference estimation with matching. A complementary analysis will be carried out on the longitudinal subsample of mothers who gave birth at least once during each of the time periods. The economic evaluation aims to capture the wider societal costs and benefits of the Salut Programme for the first 2 years of the children's lives. Incremental costs will be compared with incremental health gains and the results will be presented as a cost-consequence analysis.

Ethics and dissemination: The Regional Ethical Review Board in Umeå has given clearance for the Salut Programme research (2010-63-31M). No individual's identity will be revealed when presenting results. This study will provide information that can guide decision-makers to allocate resources optimally.
Strengths and limitations of this study

- This study protocol describes how we plan to investigate the effectiveness, costs and costeffectiveness of a universal health promotion intervention compared with care-as-usual, during pregnancy, delivery and the child's first 2 years of life.

- This is a register-based retrospective observational study that will use existing data sources, with respect to exposures and outcomes, to simulate an experiment by retrospectively identifying and comparing children and their parents in the intervention group and the nonintervention group, at two time measurement points.

- A major strength of this study is that the effectiveness of this universal health promotion programme will be observed in a real-world setting, thus increasing the external validity of the study results, and thereby increasing generalisability.

- A limitation of the study is that data on individual exposure to the Salut Programme are not available. An intention-to-treat approach is therefore used in the analysis, which implies a risk of underestimating the intervention effect.

\section{INTRODUCTION}

The populations in Europe and other wealthy parts of the world have better health now than ever before. ${ }^{1}$ However, according to a recent WHO report, this promising development in health is threatened by an increase in lifestyle-related diseases. ${ }^{1}$ Among children and adolescents, overweight, obesity and mental health problems contribute the greatest disease burden. ${ }^{2}$ Children growing up in socioeconomically disadvantaged families have worse health than others in the short and the long term. ${ }^{3}{ }^{4}$ Overweight and obesity are 
common among expectant mothers, more so among the socioeconomically disadvantaged. ${ }^{5}{ }^{6}$ Being overweight or obese is strongly associated with adverse maternal outcomes, for example, hypertensive disorders, gestational diabetes mellitus, premature delivery, instrumental delivery and caesarean section. ${ }^{7} 8$ Overweight mothers or with obesity have greater risk for adverse fetal/child outcomes such as malformations, spontaneous abortion, small-for-gestational age, large-for-gestational age, intrauterine fetal death, fetal distress and perinatal mortality and morbidity. ${ }^{7}$ Moreover, maternal pre-pregnancy overweight is associated with later childhood overweight. ${ }^{9}$ In Sweden, for example, already at 4 years of age, $17 \%$ of the boys and 22\% of the girls are overweight, and at 15 years of age, about $60 \%$ of the girls and $40 \%$ of the boys report mental health problems. ${ }^{10}$ Recognising a life course approach to health, that is, events during fetal life and childhood influence health later in life, implies that future adult health is also threatened. ${ }^{11-13}$ The so-called 'fetal programming' is explained by stimulus or damages at a critical, sensitive period of early life, and has permanent effects on the structure, physiology and metabolism, and underlying adverse disease consequences in later life. ${ }^{12} 14$ Notably, a 'healthy' parent-child relationship also reduces the risk of numerous health problems later in life. ${ }^{15}$ It is also well known that lifestyle-dependent health problems are preventable, and interventions early in the life course may have the largest long-term impact. Therefore, costeffective health promotion and preventive interventions during pregnancy and early childhood are called for, but until now the research community has failed to provide credible evidence in favour of such investments. ${ }^{16}$ A study by Robling et $a l^{17}$ evaluated the effectiveness of a homevisiting intervention 'The Family Nurse Partnership' on infant and maternal outcomes. The intervention was added to the usual health and social services in England, delivered to teenage first-time mothers. The authors found no additional short-term health benefits, but stated the necessity for the programme's longer term evaluation.

In 2005, the health authorities of the County Council of Västerbotten initiated the Salut Programme. Since $2009 / 2010$ it is a countywide ongoing universal health promotion intervention in a variety of sectors for parents and children 0-18 years, starting already during pregnancy. The Programme has been implemented since 2006 onwards, prompted by alarming reports of child overweight/obesity ${ }^{18} 19$ and trends of increased dental caries, ${ }^{20}$ already during preschool age. The Programme aims to support and strengthen initiated and ongoing health promotion interventions, and universal preventive interventions to improve health and lifestyle among expectant parents and children. One of the subaims of the Programme is that expectant mothers and new-borns are not affected by lifestyle complications during pregnancy and delivery, and that expectant mothers have a normal weight gain during pregnancy. The main focus areas are: to promote healthy eating habits, physical activity and good psychosocial health, and to prevent obesity and caries. In other words, the Programme combines both health promotion and universal prevention interventions in order to improve health and well-being, as well as to avoid ill health and disease for the whole population. ${ }^{21}$ To achieve the aforementioned aims, the Salut Programme includes a package of interventions using a family-centred approach. The interventions are integrated within ordinary public services, as described in

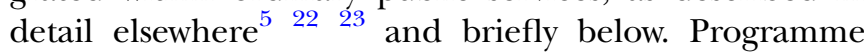
development, implementation and dissemination have been carried out stepwise, with respect to geography, which facilitates evaluation efforts.

We hypothesise that parents and children exposed to the Salut Programme, in addition to care-as-usual, will have wide-ranging health gains, such as improved health and lifestyle, at a reasonable cost, which may result in less need for healthcare. This study protocol describes how we aim to investigate the effectiveness and costeffectiveness of the Programme compared with care-as-usual, over the periods of pregnancy, delivery and the child's first 2 years of life. We cannot differentiate the effects of different components of the Salut Programme, as this is a package of interventions aimed to tackle different aspects of the population's health and lifestyle.

The study is guided by the following research questions:

1. Does the Salut Programme improve maternal and child health?

2. What are the societal costs of the Salut Programme in terms of intervention costs and societal resource use?

3. Is the Salut Programme a cost-effective public health intervention?

\section{METHODS}

\section{The study context}

Care-as-usual for expectant parents and preschool children in Sweden are tax funded hence free of charge for the public. During pregnancy, almost every woman (more than $>99 \%$ ) attends antenatal care on a regular basis, and likewise almost all preschool children and their accompanying parent attend child healthcare and dental care. Antenatal care is initiated early in pregnancy (around pregnancy week 10) and encompasses 7-9 check-ups during pregnancy, and 1 check-up postpartum. The midwife is responsible for maternal and fetal surveillance, including psychosocial and lifestyle counselling, and is supported by the doctor when needed. After the child is born, support is given through child healthcare with about 14 visits during the child's first $5^{1 / 2}$ years, and more often when needed. The child health nurse is responsible for health and development check-ups, immunisations, and psychosocial and lifestyle counselling. A doctor joins the nurse at four check-ups, usually at 6 weeks, 11/2 years, 4 years and 
$5^{1 / 2}$ years, and more often when needed. Advice on teeth brushing twice a day is given early through child healthcare, and the first invitation to dental care for check-up and health promoting advice takes place when the child is 2-3 years of age. Open preschools, attended on a drop-in basis, serve as a complement to healthcare for children not yet enrolled in regular preschools.

The Salut Programme interventions in Västerbotten County are integrated within care-as-usual and are tailored for each profession and age-adapted for the child, as described in detail elsewhere. ${ }^{5}{ }^{22-24}$ All involved staff have already taken part in several learning seminars, manuals were developed to guide everyday practice and age-specific questionnaires were developed as a basis for health counselling between professionals and children/ parents on health and lifestyle. Professionals were actively involved in intervention development and small-scale testing during the first year. Then the intervention package was decided on, and another series of learning seminars was provided during the implementation period to help the participants improve their skills, adjust their interventions, and evaluate the feasibility of the Programme. ${ }^{22}$ Previously published evaluations of the Programme have reported that practitioners significantly increased their use of a motivational interviewing approach, and collaboration between different sectors increased among other interventions. ${ }^{22}{ }^{23}$ A few examples of newly introduced interventions are given below. Before the first antenatal check-up, both the pregnant woman and her partner respond individually to a questionnaire on health and lifestyle, which guides the midwife's counselling and provides data for population health surveillance. ${ }^{22}$ Within child healthcare, fathers are encouraged to come for an individual visit when the child is about 10 months old, giving an opportunity to strengthen their role as fathers. Expectant parents are invited to free health counselling during pregnancy at the dental care, and when the child is 12-18 months of age a dental health screening is performed within child healthcare. ${ }^{22}$ Moreover, the Programme has contributed to a significant reduction in the serving of unhealthy food within open preschools. ${ }^{23}$

\section{Study design and participants}

We plan to conduct an effectiveness study and an economic evaluation study, where the Salut Programme interventions (integrated within care-as-usual) will be compared with care-as-usual over the periods of pregnancy, delivery and the child's first 2 years of life. The Salut Programme was implemented since 2006, initially only in parts of the Västerbotten County, creating the Salut area and the non-Salut area, respectively. A register-based retrospective observational study design will be used taking advantage of the already existing data sources with respect to exposures and outcomes. ${ }^{25}$ We will simulate an experiment by retrospectively identifying and comparing children and their parents in the Salut area (intervention group) and the non-Salut area (non-intervention group), and by comparing the premeasure period (2002-2004) and the postmeasure period (2006-2008) for both groups (figure 1). We will use an intention-to-treat approach; ${ }^{26}$ thus, the area of residence at the child's birth determines if the child and parents are classified as belonging to the intervention or comparison group, respectively.

More specifically, we defined the intervention group as those women (and their children) who during the year of the child's birth lived in the Salut area, that is, geographical areas within the Västerbotten County, where the Salut Programme was implemented since 2006. The nonintervention group, which corresponds to care-as-usual, is defined as those women (and their children) who during the year of the child's birth lived in the non-Salut area, that is, geographical areas within Västerbotten County where the Salut Programme was not implemented before 2009. All children in this study were born either during 20022004 or 2006-2008. The intervention group comprises 1891 children born to 1599 mothers, and the nonintervention group comprises 12723 children born to 10544 mothers. The study population and samples to be used in the different statistical analysis strategies are depicted in figure 2. The number of births, in the Salut area and the non-Salut area, during the study period determined the sample sizes. No post hoc power calculation is carried out since the only available population parameter estimates (needed for such a calculation) are based on the sample at hand. ${ }^{27} 28$

Outcomes of interest for parents and children will be observed during the premeasure period (2002-2004) and postmeasure period (2006-2008) for the intervention group and the non-intervention group. This allows us to use a difference-in-difference technique, by which we can compare the average change between the premeasure and postmeasure periods in the outcome of interest for the intervention group to the average change over time for the non-intervention group (figure 2). ${ }^{29}$ As a complementary analysis strategy, we plan to focus on a subsample of longitudinal data, that is, women who gave birth in both time periods and remained living in the same type of geographical area, the Salut area or the non-Salut area, respectively (figure 2 ).

\section{Outcome measures}

The children in the intervention group were exposed to the Salut Programme interventions during pregnancy and up to 18 months of age that had the following specific objectives: no lifestyle-related pregnancy and childbirth complications; mothers have normal weight gain during pregnancy; children have normal weight at age 18 months; and mothers reach pre-pregnancy weight by 18 months after childbirth. We have identified in the literature the most relevant indicators of the health of the child and the mother during the period around birth ${ }^{30}$ and until the child reaches 2 years of age that are available through the Umeå SIMSAM Lab. ${ }^{31}$ Thereafter, we have selected three time points in each child's and its 
Figure 1 Study design to be used in the Salut Programmes' effectiveness study and economic evaluation study. Comparisons will be made for children and their parents in the Salut area and non-Salut area, respectively, considering both the premeasure period (2002-2004) and the postmeasure period (2006-2008).
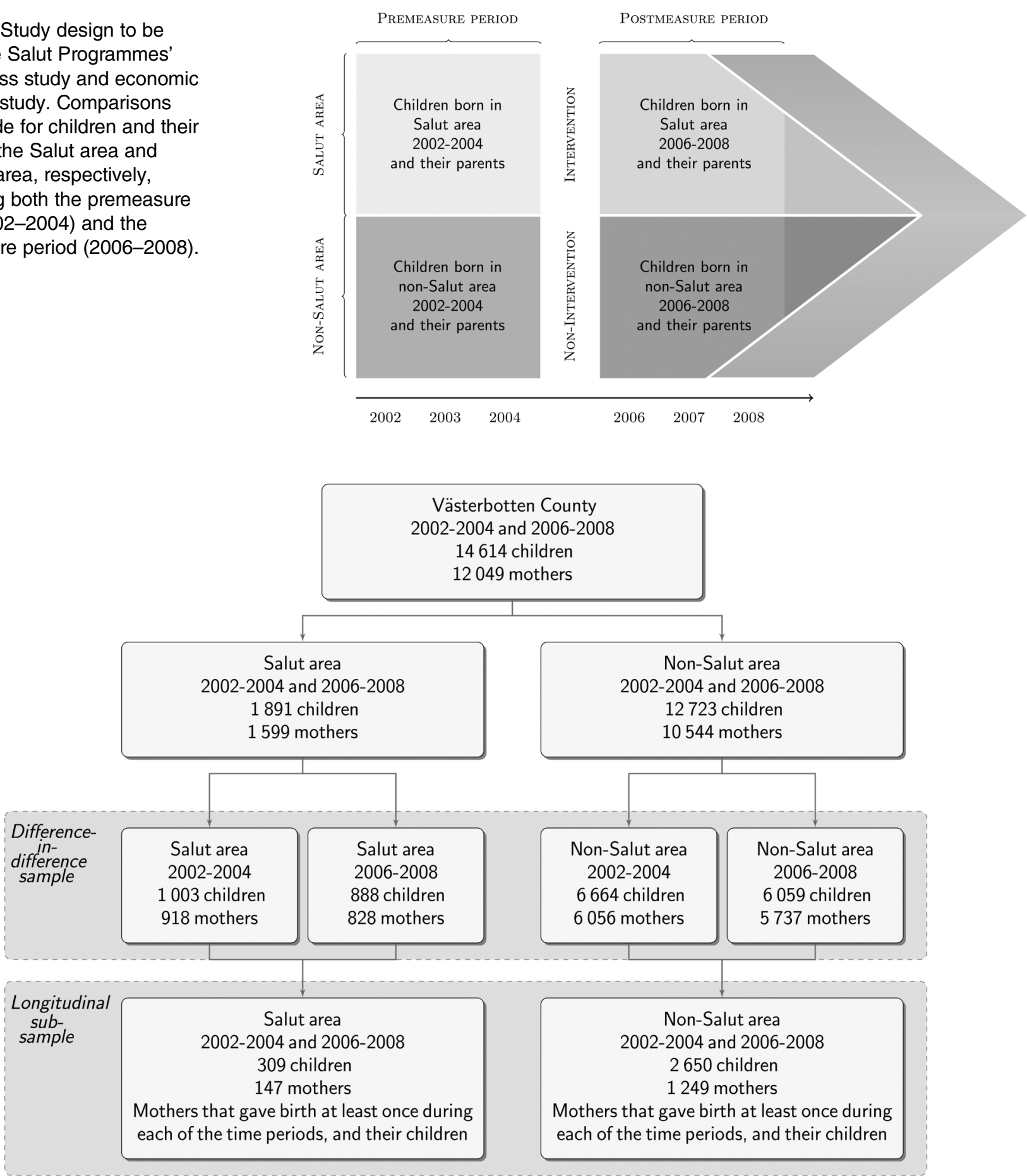

Figure 2 An overview of study population and samples to be used in the different statistical analyses strategies within the Salut Programmes' effectiveness study and economic evaluation study.

mother's life for reporting outcome measures: (1) around the child's birth - pregnancy length at delivery and any caesarean section, Apgar score (a measure of the new-born's physical condition) and weight/length immediately after the child's birth, and a child's diagnosis as either being healthy or not based on a paediatrician's examination; (2) 1 month after the child's birth - the cumulative duration of inpatient care for mother and child, respectively; and (3) 2 years after the child's birth-cumulative number of hospital visits and cumulative duration of inpatient care for mother and child, and mother's and father's cumulative social benefits for staying home from work to care for their sick child. National register sources and included variables are briefly described below and in table 1 .

\section{National registers and data access}

Sweden has a long tradition of national registers with high-quality data on health and socioeconomic 
indicators. These registers include the Swedish individual 10-digit personal identification number (ID) that follows every resident from birth (or time of immigration) to death. Within the Umeå SIMSAM Laboratory comprehensive individual-level data for the whole country's population from 1960 to 2010 has been compiled for research purposes. ${ }^{31}$ The data have been retrieved from several national registers and linked on an individual level by Statistics Sweden, and thereafter made anonymous. Furthermore, intergenerational links between parents and children have been created, by using the personal identification numbers. Our study uses data available within the Umeå SIMSAM Lab from five national registers, briefly described below.

The Multi-Generation Register comprises all individuals registered in Sweden in 1961 or later and those born in 1932 or later. Links to biological parents are present for each individual. The register is updated each year with information on individuals that migrated or were born during the year. The Register of Education includes information on the population's educational level since 1985 and is updated annually. It includes all Swedish residents aged 16-74 years and their highest level of education attained as well as year of completion. The Medical Birth Register was established in 1973 and contains information on almost all pregnancies and deliveries in Sweden, on mothers and new-borns. The National Patient Register comprises all inpatient care since 1987 including admission and discharge dates, and diagnoses for each episode. Since 2001, data on outpatient visits are available, however, not for primary care. The Swedish Social Insurance Agency's STORE data warehouse contains information on each person's annual financial benefits from the social security systems, including compensation for staying home from work to care for a sick child. The registers and variables that we plan to use in the effectiveness and the economic evaluation studies are listed in table 1 . All outcome variables will be used in the effectiveness study. The economic evaluation study will be limited to those outcomes that show a statistical significant difference between the intervention and non-intervention groups in the effectiveness study. In addition, some of the variables will be used to estimate the societal costs and benefits of the intervention, as indicated in table 1.

\section{Statistical analyses}

Descriptive statistics will be used to present characteristics and outcome variables for the intervention group and the non-intervention group at premeasure and postmeasure periods for both groups.

Data analyses will be divided in two steps. In the first step, we will use difference-in-difference estimation with matching. ${ }^{29}{ }^{32}$ This estimation strategy will account for observable differences in individual characteristics (by matching) and, under the assumption that the outcome follows parallel trends in the Salut areas and the non-Salut areas, for time-invariant unobservable differences between the intervention group and the non- intervention group. By parallel trends we mean that, without the implementation of the Salut Programme, the average change in the outcome between the premeasure period and postmeasure period would have been the same, in the Salut area and the non-Salut area. Assuming parallel trends does, however, allow for the average premeasure period outcome to differ between the two areas. Here, the mobility of individuals, that is, mothers moving from one type of area to another type of area, is not taken into account. However, during the study period the year-to-year mobility pattern in this part of Sweden is stable. ${ }^{33}$

The choice of matching variables will be governed by subject-matter knowledge on potential confounders, that is, factors that might influence the decision to live or not in a Salut area, which might affect health outcomes. Mother's age and educational level at the time of child's birth will be included as matching variables due to their significant relationship with childbirth outcomes. ${ }^{34} 35$ Depending on the type and number of matching variables, exact matching or propensity score matching will be used.

The samples that will be used are schematically presented in figure 2. Each of the 828 mothers in the intervention group (Salut area) at postmeasure (2006-2008) will be matched to one observationally similar mother in each of the other groups: the intervention group (Salut area) at premeasure (2002-2004); the non-intervention group (non-Salut area) at premeasure (2002-2004) and the non-intervention group (non-Salut area) at postmeasure (2006-2008). In this way, we will construct three matched groups such that they reflect the covariate distribution in the intervention group at postmeasure. This is done to remove biases due to differences in the distributions of age, educational level and any other matching variable between the intervention group at postmeasure and the intervention group at premeasure, the nonintervention group at premeasure and the nonintervention group at postmeasure, respectively. We will consider matching 'with replacement' allowing each mother to be used as a match more than once. In case there are several exact matches, a weighted average of these matches will be used, and in case of no exact match a mother with the closest matching variable value will be selected as a match. Using the outcome data related to the parents and the child in the intervention group at postmeasure and the three matched groups, we will estimate the average intervention effect in the intervention group by computing the mean difference-in-differences in the outcome across the intervention group and the non-intervention group. Estimates will be presented with 95\% CIs based on Abadie-Imbens $\mathrm{SEs}^{36}$ or resampling methods.

In the next step, we will conduct a longitudinal analysis, where we will restrict our attention to the subsample of women who gave birth to at least one child in each of the time periods of interest (2002-2004 and 2006-2008), and remained living in the same type of geographical area over the whole period (2002-2008). The subsample of 
Table 1 National registers and variables to be used in the Salut Programme's effectiveness study and economic evaluation study

\begin{tabular}{|c|c|c|c|}
\hline National registers & Variables & $\begin{array}{l}\text { Effectiveness } \\
\text { study }\end{array}$ & $\begin{array}{l}\text { Economic } \\
\text { evaluation study }\end{array}$ \\
\hline \multicolumn{4}{|l|}{ Demographic characteristics } \\
\hline \multirow[t]{8}{*}{ Multigeneration register* } & Child's ID & & \\
\hline & Child's date of birth & & \\
\hline & Child's place of birth (parish) & & \\
\hline & Mother's ID & & \\
\hline & Mother's date of birth & & \\
\hline & Father's ID & & \\
\hline & Child born in Salut area vs non-Salut area & & \\
\hline & $\begin{array}{l}\text { Premeasure (2004-2006) or postmeasure } \\
(2006-2008)\end{array}$ & & \\
\hline The register of education* & $\begin{array}{l}\text { Mother's educational level at child's year of } \\
\text { birth }\end{array}$ & & \\
\hline \multicolumn{4}{|l|}{ Outcomes around child's birth } \\
\hline \multirow[t]{11}{*}{ The medical birth registert } & Mother's weight at first antenatal visit & $\sqrt{ }$ & \\
\hline & Mother's height & $\sqrt{ }$ & \\
\hline & $\begin{array}{l}\text { Mother's smoking status at first antenatal } \\
\text { visit }\end{array}$ & $\sqrt{ }$ & \\
\hline & Pregnancy length at delivery & $\sqrt{ }$ & \\
\hline & Delivery ends with caesarean section & $\sqrt{ }$ & \\
\hline & Apgar score $\neq 1,5$ and 10 min after delivery & $\sqrt{ }$ & \\
\hline & Child's length at birth & $\sqrt{ }$ & \\
\hline & Child's weight at birth & $\sqrt{ }$ & \\
\hline & $\begin{array}{l}\text { Low child weight relative to pregnancy } \\
\text { length }\end{array}$ & $\sqrt{ }$ & \\
\hline & $\begin{array}{l}\text { High child weight relative to pregnancy } \\
\text { length }\end{array}$ & $\sqrt{ }$ & \\
\hline & Healthy child diagnosis & $\sqrt{ }$ & \\
\hline National patient registert & $\begin{array}{l}\text { Mother's duration of inpatient care related to } \\
\text { delivery of the child }\end{array}$ & $\sqrt{ }$ & $\sqrt{ }$ \\
\hline \multicolumn{4}{|c|}{ Outcomes during the first month after child's birth } \\
\hline \multirow[t]{2}{*}{ National patient register } & Child's cumulative duration of inpatient care & $\sqrt{ }$ & $\sqrt{ }$ \\
\hline & $\begin{array}{l}\text { Mother's cumulative duration of inpatient } \\
\text { care }\end{array}$ & $\sqrt{ }$ & $\sqrt{ }$ \\
\hline \multicolumn{4}{|c|}{ Outcomes during the first 2 years after child's birth } \\
\hline \multirow[t]{4}{*}{ National patient register } & Child's cumulative duration of inpatient care & $\sqrt{ }$ & $\sqrt{ }$ \\
\hline & $\begin{array}{l}\text { Child's cumulative number of outpatient } \\
\text { care visits }\end{array}$ & $\sqrt{ }$ & $\sqrt{ }$ \\
\hline & $\begin{array}{l}\text { Mother's cumulative duration of inpatient } \\
\text { care }\end{array}$ & $\sqrt{ }$ & $\sqrt{ }$ \\
\hline & $\begin{array}{l}\text { Mother's cumulative number of outpatient } \\
\text { care visits }\end{array}$ & $\sqrt{ }$ & $\sqrt{ }$ \\
\hline $\begin{array}{l}\text { The Swedish Social Insurance } \\
\text { Agency's STORE data warehouse* }^{*}\end{array}$ & $\begin{array}{l}\text { Mother's and father's cumulative number of } \\
\text { days for care of sick child }\end{array}$ & $\sqrt{ }$ & $\sqrt{ }$ \\
\hline
\end{tabular}

\section{*Held by Statistics Sweden.}

†Held by the National Board of Health and Welfare.

$\ddagger$ Apgar score: a measure of the new-born’s physical condition.

women that remained living in the Salut area belongs to the intervention subgroup, whereas the subsample of women that remained living in the non-Salut area belongs to the non-intervention subgroup. A simple matching estimator will be used to estimate the average intervention effect in the intervention subgroup compared with the non-intervention subgroup. Measures from the preintervention period (2002-2004) will be considered as baseline values, and each mother in the intervention subgroup will be matched to an observationally similar mother in the non-intervention subgroup. Matching will be done based on the following potential confounding variables, mother's age and mother's educational level. Replacement of one or several matches will be handled in the same way as in the differencein-difference analysis.

Using outcome data from the postmeasure period related to the parents and the child in the intervention 
subgroup and in the matched group, we will estimate the average intervention effect in the intervention group for the longitudinal subsample by computing the mean difference in the postmeasure period outcome between the intervention subgroup and the matched group. Estimates will be presented with $95 \%$ CIs based on Abadie-Imbens SEs. ${ }^{36}$

\section{Economic evaluation}

The economic analysis will be performed from a societal perspective, that is, we will seek to capture all societal costs and benefits as fully as possible. ${ }^{37}$

Costs to deliver the Salut Programme will be estimated based on the Salut Programme's process management group's records concerning professionals' time to participate in educational seminars related to the Programme and their time needed to deliver the interventions, costs for materials handed out to participants, the opportunity cost for parents' time spent on the intervention, etc.

Direct costs will be derived from information on the use of other healthcare resources outside the Salut Programme such as maternal inpatient care related to delivery, and children's and mothers' inpatient and outpatient care due to illness until the child reaches 3 years of age. Maternal inpatient care related to delivery will be valued using unit cost-estimates from Diagnostic Related Group Codes available from the Swedish National Board of Health and Welfare. Children's and mother's inpatient and outpatient care due to illness will be valued using unit cost-estimates from the Cost per Patient Database from the Swedish Association of Local Authorities and Regions.

Productivity losses due to illness of mothers and children will be included in the analysis using information on mothers' inpatient and outpatient care, and parental leave taken to care for a sick child (by mothers and fathers). All costs will be calculated for the first 3 years after the child's birth. The economic evaluation will be presented as a cost-consequence analysis, ${ }^{37}$ where all costs and significant outcomes will be listed separately, which allows policymakers to compare the incremental costs with the incremental health gains of the Salut Programme. We will explore the possibilities of conducting a long-term cost-effectiveness analysis using economic decision modelling based on the outcomes that are deemed significant and relevant to predict future cost-savings and health gains from implementing the Salut Programme on the target population.

\section{DISCUSSION}

Health problems during childhood are a concern in many parts of the world, including wealthy societies, ${ }^{38}$ and health inequity between socioeconomic groups is increasing over time. ${ }^{39}$ Taking a life course perspective on health and disease implies that health and economic gains will be larger the earlier in life effective health promotion actions are adopted. ${ }^{40}$ However, to the best of our knowledge, there is no convincing scientific evidence for any universal complex intervention starting early in life to be both effective and cost-effective, but efforts to address this need are in progress by the scientific community. ${ }^{41}$

This study protocol describes how we plan to investigate the effectiveness, costs and cost-effectiveness of the Salut Programme, integrated into routine practice, compared with care-as-usual, in the Västerbotten County Council in Sweden, during pregnancy, delivery and the child's first 2 years of life. We hypothesise that parents and children exposed to the Salut Programme will enjoy wide-ranging health gains at a reasonable cost, compared with care-as-usual. This study addresses the need for comprehensive evaluations of universal health promotion programmes integrated within ordinary public services. The results will be highly relevant for universal health promotion efforts, and increase the capacity of policy and decision-makers to assess and apply research findings in decision-making by providing them with evidence-based research.

A major strength of this study is that the effectiveness of this universal health promotion programme will be observed in a real-world setting. This allows assessing the impact of the interventions on individuals with a varied range of characteristics, thus increasing the external validity of the study results, and thereby increasing generalisability. Another strength is that the Umeå SIMSAM Lab provides a rich source of individual-level data on demography, socioeconomic indicators and health, and importantly intergenerational links allowing one to identify each child's parents, and geographical coordinates for every individual's residential area. ${ }^{31}$ We believe that where such data are available, researchers have a duty to use it. In addition, the rich data allow for the use of statistical techniques that do not require strong assumptions regarding the data generating mechanisms.

However, there are some methodological challenges that need to be addressed. A limitation of the study is that data on individual exposure to the Salut Programme are not available. Therefore, an intention-to-treat approach will be used in the analysis, ${ }^{26}$ which implies a risk of underestimating the intervention effect. The main reason is that the Salut Programme is not a research study but an ongoing intervention implemented by the Västerbotten County Council. Although future research was anticipated at the time (2005-2006), reflected in the stepwise implementation design, data on individual exposure to different elements of the Programme have not been collected, as might be expected of a research study. In addition, as is typical of universal and complex public health interventions, it is difficult (or even impossible) to secure full delivery of the intervention on an individual level. Providers make choices regarding how they integrate the Programme into routine practice, and parents make choices regarding their use of services such as whether or not to attend open preschools. While this is a limitation compared with clinical trials, integration of 
the Programme into routine practice has enabled the sustainability of the Programme beyond the current study period. $^{22} 23$

Another methodological challenge is that although the Salut and non-Salut areas are both within the Västerbotten County, population characteristics may differ, which carries the risk of introducing selection bias. We plan to control this in the analyses by using matching, thus accounting for potential confounders. However, we are aware of the risk for residual confounding; thus, it is unlikely that we will be able to take all confounders fully into account. Despite the rich source of existing national register data available for this study, there are data limitations that hamper the analyses, which is evident from this study protocol. We do not have access to, for example, breastfeeding duration, 2 years old's weight and height or quality of life data, or data on the full resource use by target group, such as primary healthcare visits or medication used. This may contribute to uncertainty of the results from the economic evaluation.

Universal complex interventions implemented in reallife settings such as the Salut Programme are scarce and pose large challenges with respect to implementation, dissemination and evaluation. ${ }^{42}$ Observational studies offer a credible solution to fill in the gaps left by randomised controlled trials that often have financial, ethical and time constraints. This study will apply novel methods to the evaluation of public health interventions, including cost-effectiveness analyses, and thereby provide information that can guide decision-makers to allocate resources optimally.

Acknowledgements The authors would like to acknowledge all parents in Västerbotten County who took part in the Salut Programme, as well as all the healthcare professionals involved in the implementation and delivery of the Programme. The Umeå SIMSAM Lab data infrastructure used in this study was developed with support from the Swedish Research Council and by strategic funds from Umeå University.

Contributors Al and IF conceived and designed the study. Al, EE and ML constitute the scientific steering group for the Salut Programme, and Al is the principal investigator for the Umeå SIMSAM Lab, both pre-requisites for the present study. A detailed plan for the statistical analyses was made by $\mathrm{JH}$, and the plan for the economic evaluation was made by IF, A-MP-B and FS. All the authors (IF, EE, JH, FS, ML, A-MP-B and Al) contributed to the writing process and have approved the final manuscript.

Funding This study was funded by the Swedish Research Council for Health, Working Life and Welfare (FORTE), grant number 2014-1399.

Competing interests None declared.

Ethics approval The Regional Ethical Review Board in Umeå has given clearance for the Salut Programme research (2010-63-31M) and for the Umeå SIMSAM Lab research (2010-157-310), including the present study. Within the Umeå SIMSAM Lab, rigorous measures are taken to protect personal integrity, and when presenting results, no individual's identity will be revealed. The Salut Programme is not expected to cause any harm to either children or parents during or after pregnancy. Results will be presented both in the form of peer-reviewed journal articles, and also directly communicated to healthcare decision-makers in the Västerbotten County and at a national level. This study will provide information that can guide decision-makers to allocate resources optimally.
Provenance and peer review Not commissioned; externally peer reviewed.

Data sharing statement Additional data are available from the corresponding author.

Open Access This is an Open Access article distributed in accordance with the Creative Commons Attribution Non Commercial (CC BY-NC 4.0) license, which permits others to distribute, remix, adapt, build upon this work noncommercially, and license their derivative works on different terms, provided the original work is properly cited and the use is non-commercial. See: http:// creativecommons.org/licenses/by-nc/4.0/

\section{REFERENCES}

1. World Health Organization. World Health Statistics 2015. Geneva, Switzerland: World Health Organization, 2015:162.

2. Mathers $C D$, Loncar $D$. Projections of global mortality and burden of disease from 2002 to 2030. PLoS Med 2006;3:e442.

3. Gupta RP, de Wit ML, McKeown D. The impact of poverty on the current and future health status of children. Paediatr Child Health 2007;12:667-72.

4. Melchior M, Moffitt TE, Milne BJ, et al. Why do children from socioeconomically disadvantaged families suffer from poor health when they reach adulthood? A life-course study. Am J Epidemiol 2007; 166:966-74.

5. Eurenius $\mathrm{E}$, Lindkvist $\mathrm{M}$, Sundqvist $\mathrm{M}$, et al. Maternal and paternal self-rated health and BMI in relation to lifestyle in early pregnancy: the Salut Programme in Sweden. Scand J Public Health 2011;39:730-41.

6. Edvardsson K, Lindkvist M, Eurenius E, et al. A population-based study of overweight and obesity in expectant parents: socio-demographic patterns and within-couple associations. BMC Public Health 2013;13:923.

7. Cedergren M. Effects of gestational weight gain and body mass index on obstetric outcome in Sweden. Int $J$ Gynaecol Obstet 2006:93:269-74

8. Linné Y. Effects of obesity on women's reproduction and complications during pregnancy. Obes Rev 2004;5:137-43.

9. Weng SF, Redsell SA, Swift JA, et al. Systematic review and meta-analyses of risk factors for childhood overweight identifiable during infancy. Arch Dis Child 2012;97:1019-26.

10. Socialstyrelsen [The National Board of Health and Welfare]. Barns och ungas hälsa, vård och omsorg 2013 [Children and young people's health and healthcare in 2013]. Stockholm: Socialstyrelsen [The National Board of Health and Welfare], 2013.

11. Ben-Shlomo Y, Kuh D. A life course approach to chronic disease epidemiology: conceptual models, empirical challenges and interdisciplinary perspectives. Int J Epidemiol 2002;31:285-93.

12. Gicquel C, El-Osta A, Le Bouc Y. Epigenetic regulation and fetal programming. Best Pract Res Clin Endocrinol Metab 2008;22:1-16.

13. McMillen IC, Robinson JS. Developmental origins of the metabolic syndrome: prediction, plasticity, and programming. Physiol Rev 2005;85:571-633

14. Lau C, Rogers JM. Embryonic and fetal programming of physiological disorders in adulthood. Birth Defects Res C Embryo Today 2004;72:300-12.

15. Stewart-Brown S. Improving parenting: the why and the how. Arch Dis Child 2008;93:102-4.

16. Batura N, Hill Z, Haghparast-Bidgoli $\mathrm{H}$, et al. Highlighting the evidence gap: how cost-effective are interventions to improve early childhood nutrition and development? Health Policy Plan 2015;30:813-21.

17. Robling M, Bekkers MJ, Bell K, et al. Effectiveness of a nurse-led intensive home-visitation programme for first-time teenage mothers (Building Blocks): a pragmatic randomised controlled trial. Lancet 2016;387:146-55.

18. Bergström E, Blomquist $\mathrm{HK}$. Is the prevalence of overweight and obesity declining among 4-year-old Swedish children? Acta Paediatr 2009;98:1956-8.

19. Blomquist HK, Bergström E. Obesity in 4-year-old children more prevalent in girls and in municipalities with a low socioeconomic level. Acta Paediatr 2007;96:113-16.

20. Stecksén-Blicks C, Holgerson PL, Twetman S. Caries risk profiles in two-year-old children from northern Sweden. Oral Health Prev Dent 2007;5:215-21.

21. World Health Organization. Prevention and promotion in mental health. Geneva: World Health Organization, 2002:46.

22. Edvardsson K, Garvare R, Ivarsson A, et al. Sustainable practice change: professionals' experiences with a multisectoral child health promotion programme in Sweden. BMC Health Serv Res 2011;11:61. 
23. Edvardsson $\mathrm{K}$, Ivarsson A, Garvare $\mathrm{R}$, et al. Improving child health promotion practices in multiple sectors-outcomes of the Swedish Salut Programme. BMC Public Health 2012;12:920.

24. Höög E, Garvare R, Ivarsson A, et al. Challenges in managing a multi-sectoral health promotion program. Leadersh Health Serv 2013;26:368-86.

25. Berger ML, Martin BC, Husereau D, et al. A questionnaire to assess the relevance and credibility of observational studies to inform health care decision making: an ISPOR-AMCP-NPC Good Practice Task Force report. Value Health 2014;17:143-56.

26. Gupta SK. Intention-to-treat concept: a review. Perspect Clin Res 2011;2:109-12.

27. Vandenbroucke JP, von Elm E, Altman DG, et al. Strengthening the Reporting of Observational Studies in Epidemiology (STROBE): explanation and elaboration. PLoS Med 2007:4:e297.

28. Schulz KF, Grimes DA. Sample size calculations in randomised trials: mandatory and mystical. Lancet 2005;365:1348-53.

29. Lechner M. Estimation of causal effects by difference-in-difference methods. 3rd edn. Now Publishers, 2011.

30. Escuriet $\mathrm{R}$, White $\mathrm{J}$, Beeckman $\mathrm{K}$, et al. Assessing the performance of maternity care in Europe: a critical exploration of tools and indicators. BMC Health Serv Res 2015;15:491.

31. Lindgren U, Nilsson $\mathrm{K}$, de Luna $\mathrm{X}$, et al. Data resource profile: Swedish Microdata Research from Childhood into Lifelong Health and Welfare (Umeå SIMSAM Lab). Int J Epidemiol 2016. [Epub ahead of print 11 May 2016]. doi:10.1093/ije/dyv358

32. Imbens GW, NBER Working Papers-York University., National Bureau of Economic Research. Nonparametric estimation of average treatment effects under exogeneity: a review. NBER technical working paper series no t0294. Cambridge, Mass: National Bureau of Economic Research, 2003.

33. Statistics Sweden. Migration by region, age and sex. Year 19972015. Secondary Migration by region, age and sex. Year 1997-
2015. http://www.statistikdatabasen.scb.se/pxweb/sv/ssd/START BE BE0101 BE0101J/Flyttningar97/?

rxid=53f37737-938a-4473-a723-d972a142d99e

34. Härkönen J, Kaymakçalan H, Mäki P, et al. Prenatal health, educational attainment, and intergenerational inequality: the Northern Finland Birth Cohort 1966 Study. Demography 2012;49:525-52.

35. Chiavarini M, Bartolucci F Gili A et al. Effects of individual and social factors on preterm birth and low birth weight: empirical evidence from regional data in Italy. Int $J$ Public Health 2012;57:261-8.

36. Abadie A, Imbens GW. Large sample properties of matching estimators for average treatment effects. Econometrica 2006;74:235-67.

37. Drummond M, Sculpher M, Torrance G, et al. Methods for the economic evaluation of health care programmes. 3rd edn. Oxford: Oxford University Press, 2005.

38. Braveman P. What is health equity: and how does a life-course approach take us further toward it? Matern Child Health $J$ 2014;18:366-72.

39. Closing the Gap in a Generation: Health Equity through Action on the Social Determinants of Health. Secondary Closing the Gap in a Generation: Health Equity through Action on the Social Determinants of Health. http://www.who.int/social_determinants/ thecommission/finalreport/en/

40. Barnett WS. Effectiveness of early educational intervention. Science 2011;333:975-8.

41. Helland SH, Bere E, Øverby NC. Study protocol for a multi-component kindergarten-based intervention to promote healthy diets in toddlers: a cluster randomized trial. BMC Public Health 2016;16:273.

42. Faria $\mathrm{R}$, Weatherly $\mathrm{H}$, Kiss $\mathrm{N}$, et al. The challenges in evaluating the cost-effectiveness of complex interventions. Value Health 2015;18: A727-8. 